\section{A word processing-linked FORTRAN data base of journal and book references}

\author{
ALICE J. O'TOOLE \\ Catholic University of America, Washington, $D C$
}

A FORTRAN program for the creation and maintenance of a direct-access file of journal and book references is described. References stored in the file can be accessed by an access number, an author name, or a keyword and written to a user-designated "runoff file" in American Psychological Association (APA) format. Such a data base can be kept as a library of references to be accessed for a bibliography or literature compilation.

The problem of maintaining an organized file of journal and book references is common to all researchers. Index card files are often time-consuming to maintain and have become outdated in research laboratories where papers and proposals are typically prepared using word processing systems on a laboratory computer. The purpose of this program is to simplify the task of preparing a paper or proposal by making use of an active data base of journal and book references in connection with a word processing system.

\section{Implementation Information}

The program is written in FORTRAN IV to operate on a Digital Equipment Corporation (DEC) PDP-11/23 minicomputer under the RSX-11M operating system. Currently, the program is used on a DEC VAX $11 / 780$ computer with a VMS operating system. The "runoff" program supported by many DEC operating systems is required to derive full benefit from the access routines. However, users with some knowledge of FORTRAN can easily alter the output format statements to suit other text processing systems.

The data base resides on disk and consists of an alphabetically maintained linked list of references connected by "integer-implemented" pointers. This type of structure allows for efficient use of the FORTRAN directaccess files. The executable code occupies 69 blocks of memory.

\section{Program Features}

Entry. Two entry options are provided in the main program menu. The "create and enter" option creates a new direct-access file and allows the user to enter references, whereas the "enter" option opens an existing file for the entry of more references.

The author gratefully acknowledges the contributions of James $\mathbf{H}$. Howard, Jr., at all stages of the project and the comments of Kevin Bennett and Janet McLeod on an earlier verison of the manuscript. The author's current mailing address is: Department of Psychology, Brown University, Box 1853, Providence, RI 02912.
Both modes of entry prompt the user for the type of reference to be entered. If the code for "journal article" is specified, the program prompts for number of authors, authors' names, article title, journal, year, volume, page numbers, and two keywords. If the code for "'book" is specified, the user must supply the number of authors, authors' names, title, publisher, city, year, and two keywords. For book entries, the volume number and page prompts should be ignored.

When a complete reference has been entered, it is assigned an access number, which is displayed on the terminal screen. A listing of these numbers along with authors' names and publication year for all references currently in the file may be obtained by the "get listing" option provided.

Access. Two access options are provided by the main program menu. Direct access retrieves the records by direct-access number. Sequential access retrieves references by either author name or keyword.

The access routines write the references in the runoff program format to a file specified by the user. When "documented" using the runoff program, the file becomes an APA-formatted listing of the references accessed.

If the direct-access option is chosen, the program prompts for the number of references to be acccessed and then prompts for one access number at a time. When all the references to be retrieved have been accessed, the user is offered the option of alphabetizing the references before they are written to an output file.

The references are written to the designated file in runoff format. The runoff file may be documented and printed out as an APA-formatted bibliography or may be merged with an existing runoff file and then documented.

If the sequential-access option is chosen, the user is given the choice of searching by author name or by keyword. The program prompts for a search word and then retrieves all references with a search-field and a searchword match. For example, if " Smith, J. K." is specified in an author sequential search, the program retrieves all references in which J. K. Smith is an author. If there are no articles or books by J. K. Smith in the file, then "None found" will appear on the screen.

Sequential keyword access is identical to author search but with the search occurring by keyword. Articles retrieved by either mode of sequential search are written to the file in alphabetical order and may be documented in the way described for direct-access references.

Delete. The "delete" option prompts the user for an access number. The specified record is deleted from the "linked list" of references, and the record number associated with the deleted reference is then stored on an "empty" stack maintained in the first record of the file. These record numbers are reused as new references are entered into the file.

Change. Typing or spelling errors may be corrected 
by using the "change" option. When this option is specified, the user is prompted for the access number of the reference to be changed. A second prompt asks for the number of fields to be changed. Next, a menu appears with the list of all changeable fields. The program prompts for one field code at a time until all of the fields to be changed have been specified.

Prompts then appear for each field specified, and the user is asked to enter the corrected version of the field. Author fields may not be changed with this routine. If there is an error in any of the author fields, then the reference must be deleted and reentered.

Extend. The "extend" option allows the user to extend the size of the direct-access file by a specified number of blocks. Since the program provides for an initial allocation of 100 blocks for the data base, the user should need this option only for large bibliographic files (approximately 100 references).

Listing. A "get listing" option is provided in the main program menu. This produces an alphabetized listing of all references in the file by access number, author(s), and publication year. The listing is then output to a userdesignated runoff file for documentation.

APA-Formatted Listing. This option outputs a complete APA-formatted alphabetized listing of all references in the file to a user-designated runoff file.

\section{Advantages and Limitations}

One major advantage of this program over others that depend primarily on sequential types of access (e.g., Glass, Kaetzel, \& Smith, 1980), is that direct access, in general, minimizes retrieval time. Access time for the direct-access operation is also less affected by variables such as the sorted position of the record in the file.

Alphabetization of references retrieved by the directaccess operation, however, is slightly more timeconsuming. Since alphabetization in the file is achieved and maintained by means of "integer-implemented" pointers, sequential search by author or keyword automatically produces an alphabetized listing of the refer- ences. For this reason, the sequential-access time is sometimes comparable to alphabetized direct-access time.

The speed of alphabetizing references as they are first entered into the file is aided by the maintenance of a 26element array stored in the first record of the file. This array contains the direct-access number of the first record alphabetically of each letter of the alphabet. With the maintenance of this array, search time for the proper alphabetical position of each record is reduced appreciably. Thus, if the name of the first author of a record to be entered begins with " $S$," the search for the alphabetical position of the record can begin with the first " $S$ " record. The speed advantage of this type of system increases with the number of records in the file.

The data base that is created and maintained by this program resides on disk. Thus, very large bibliographic files can be assembled. The size of the data base, then, is constrained by the amount of disk storage available and not by the amount of main memory available.

The program is limited in some ways by the constraints of the formatting. Occasionally, references have unusually long titles or a large number of authors. This program limits titles to 160 characters and the number of authors to eight. Although these limits are sufficient for most references, they do not accommodate all. When these types of difficulties occur, simple editing of the output runoff file can usually rectify the situation.

\section{Availability}

A listing of the source code and a floppy disk copy are available from the author at cost. A more detailed version of this manuscript, including entry and access examples, will be provided upon request.

\section{REFERENCE}

Glass, R. A., Kaetzel, L. J., \& SMith, G. R. (1980). A computer data base system for indexing research papers. Behavior Research Methods \& Instrumentation, 12, 547-548.

(Manuscript accepted for publication September 21, 1984.) 